\title{
PLANARIZATIONS AND MAPS TAKING LINES TO LINEAR WEBS OF CONICS
}

\author{
VLADLEN TIMORIN
}

\begin{abstract}
Aiming at a generalization of a classical theorem of Möbius, we study maps that take line intervals to plane curves, and also maps that take line intervals to conics from certain linear systems.
\end{abstract}

\section{Introduction}

1.1. The problem. In 1827, Möbius [5] proved that a continuous one-to-one map $\mathbb{R} P^{2} \rightarrow \mathbb{R} P^{2}$ that takes any straight line to a straight line is a projective transformation. As was noted later by von Staudt [12], the continuity assumption was superfluous.

Möbius used the term collineation for a map that takes collinear points to collinear points. More precisely, let $U \subset \mathbb{R} P^{2}$ be an open subset. A map $F: U \rightarrow \mathbb{R} P^{n}$ is called a collineation if there exists an open set of lines $L \subset \mathbb{R} P^{2}$ such that $L \cap U \neq \varnothing$, and the set $F(U \cap L)$ is collinear, i.e., lies in a projective line. A very minor modification of Möbius' original argument yields the following theorem. Any continuous collineation $F: U \rightarrow \mathbb{R} P^{n}$ is a restriction of a projective embedding, or a map to a subset of a projective line, possibly after replacing $U$ with a smaller open set.

By analogy, we define a planarization as a map $F: U \rightarrow \mathbb{R} P^{n}$, for which there exists an open set of lines $L \subset \mathbb{R} P^{2}$ such that $U \cap L \neq \varnothing$, and the set $F(U \cap L)$ lies in a projective hyperplane. We consider the following problem: describe all planarizations $F: U \rightarrow \mathbb{R} P^{n}$. This problem is a direct generalization of the problem considered by Möbius.

On the other hand, it is motivated by the following class of problems. Let $\mathcal{L}$ be a linear system of algebraic curves in $\mathbb{R} P^{2}$, and $U \subset \mathbb{R} P^{2}$ an open set. We say that a map $f: U \rightarrow \mathbb{R} P^{2}$ takes all lines to $\mathcal{L}$-curves if $f(U \cap L)$ belongs to a curve from $\mathcal{L}$ for every line $L \subset \mathbb{R} P^{2}$. For various linear systems $\mathcal{L}$, we would like to know all sufficiently smooth maps that take lines to $\mathcal{L}$-curves. In the case, where $\mathcal{L}$ consists of all circles, this problem came from Nomography (see e.g., [4]); it was solved by Khovanskii [3]. Maps taking lines to circles have also been investigated in higher dimensions $[2,6,7]$. Large classes of such maps are provided by generalized Hopf fibrations [9] and, more generally, by quadratic rational parameterizations of spheres [8].

If a sufficiently smooth map $f: U \rightarrow \mathbb{R} P^{2}$ takes lines to $\mathcal{L}$-curves, then it gives rise to a planarization $F=\Phi_{\mathcal{L}} \circ f: U \rightarrow \mathbb{R} P^{n}$, where $\Phi_{\mathcal{L}}: \mathbb{R} P^{2} \rightarrow \mathbb{R} P^{n}$ is the rational map, whose homogeneous components are generating equations for $\mathcal{L}$ (more details are given below). This was my original motivation for studying planarizations.

Received by the editors August 8, 2011. 
1.2. Examples of planarizations. Let us now give some examples of planarizations.

Trivial planarizations. A trivial planarization is a map $F: U \rightarrow \mathbb{R} P^{n}$, whose image lies in some hyperplane.

Co-trivial planarizations. A planarization $F: U \rightarrow \mathbb{R} P^{n}$ is co-trivial if there is a point $o \in \mathbb{R} P^{n}$ such that, for some open set of lines $L \subset \mathbb{R} P^{2}$, the set $F(U \cap L)$ is contained in a hyperplane passing through $o$. An example of a co-trivial planarization can be constructed as follows. Consider any planarization $G: U \rightarrow \mathbb{R}^{n-1}$, e.g., a projective map. Also consider any continuous function $\phi: U \rightarrow \mathbb{R}$. Then we can define the map $F: U \rightarrow \mathbb{R}^{n}=\mathbb{R}^{n-1} \times \mathbb{R}$ by the formula $F(u)=(G(u), \phi(u))$. This is a co-trivial planarization (the corresponding point $o$ is $(0, \infty)$, i.e., the intersection of the projective closure of $0 \times \mathbb{R}$ with the hyperplane at infinity).

Rational degree $d$ maps. A rational degree $d$ map is a rational map $\mathbb{R} P^{2}-\rightarrow \mathbb{R} P^{n}$ given in homogeneous coordinates by homogeneous degree $d$ polynomials without a nonconstant common polynomial factor. A rational degree $d$ map can have some points of indeterminacy, where all defining polynomials vanish simultaneously. Any rational degree $d$ map $\mathbb{R} P^{2} \rightarrow \mathbb{R} P^{n}$ with $n>d$ is a planarization. To prove this statement, it suffices to show that the image of every rational degree $d$ map $\mathbb{R} P^{1} \rightarrow \mathbb{R} P^{n}$ is contained in a $d$-dimensional projective subspace. Let $\left[u_{0}: u_{1}\right]$ be homogeneous coordinates in $\mathbb{R} P^{1}$. The lift of a rational degree $d$ map $Q: \mathbb{R} P^{1} \rightarrow \mathbb{R} P^{n}$ to $\mathbb{R}^{n+1}$ has the form

$$
\tilde{Q}\left(u_{0}, u_{1}\right)=u_{0}^{d} A_{0}+u_{0}^{d-1} u_{1} A_{1}+\cdots+u_{1}^{d} A_{d}
$$

where $A_{0}, \ldots, A_{d}$ are some constant vectors in $\mathbb{R}^{n+1}$. Hence the image of $Q$ is contained in the projectivization of the subspace spanned by $A_{0}, \ldots, A_{d}$. This projective subspace is at most $d$-dimensional.

Duality. Projective duality between points and hyperplanes yields a remarkable duality on planarizations. Recall that points $\hat{x}$ in $\mathbb{R} P^{n *}$ correspond to hyperplanes $P_{\hat{x}}$ in $\mathbb{R} P^{n}$. In particular, points $\hat{a} \in \mathbb{R} P^{2 *}$ correspond to lines $L_{\hat{a}} \subset \mathbb{R} P^{2}$, and points $a \in \mathbb{R} P^{2}$ correspond to lines $L_{a} \subset \mathbb{R} P^{2 *}$. Let $U \subset \mathbb{R} P^{2}$ be an open set, and $F: U \rightarrow$ $\mathbb{R} P^{n}$ a planarization. Then we can define the dual planarization $\hat{F}: \hat{U} \rightarrow \mathbb{R} P^{n *}$ as follows. By definition, the set $\hat{U} \subset \mathbb{R} P^{2 *}$ is the set of all points $\hat{a} \in \mathbb{R} P^{2 *}$ such that $F\left(U \cap L_{\hat{a}}\right)$ lies in a unique hyperplane $P_{\hat{x}}$. We set $\hat{F}(\hat{a})=\hat{x}$. Thus, we defined a map $\hat{F}: \hat{U} \rightarrow \mathbb{R} P^{n *}$. We need to prove that this map is a planarization: for every $a \in U$, the set $\hat{F}\left(\hat{U} \cap L_{a}\right)$ is contained in a hyperplane. Indeed, the set $\hat{F}\left(\hat{U} \cap L_{a}\right)$ consists of points $\hat{x}$ such that $P_{\hat{x}}$ contains the set $F(U \cap L)$ for some line $L$ passing through $a$. But these hyperplanes $P_{\hat{x}}$ contain the point $F(a)$. Therefore, all corresponding points $\hat{x}$ lie in the hyperplane $P_{F(a)}$. Note that the set $\hat{U}$ may be empty. In this case, we say that the dual of $F$ is empty. If $\hat{F}$ is nonempty, and its dual is also nonempty, then the dual of $\hat{F}$ coincides with $F$ on some open subset of $U$, as follows easily from the preceding discussion.

Duality gives rise to more examples of planarizations. E.g., we can consider dual planarizations to quadratic rational maps $U \rightarrow \mathbb{R} P^{3}$. These dual planarizations are not, in general, quadratic rational maps. They are cubic; although not immediately obvious, it can be verified by direct computation. 
1.3. Main results. The main result of this paper is the following theorem about planarizations with values in $\mathbb{R} P^{3}$ (and its applications to maps that take lines to conics):

Theorem 1.1. Let $F: U \rightarrow \mathbb{R} P^{3}$ be a sufficiently smooth planarization. Then there exists an open subset $U^{\prime} \subset U$ such that at least one of the following holds:

- the map $\left.F\right|_{U^{\prime}}$ is a trivial planarization;

- the map $\left.F\right|_{U^{\prime}}$ is a co-trivial planarization;

- the map $\left.F\right|_{U^{\prime}}$ is a rational map of degree at most 3.

Not all rational maps $F: \mathbb{R} P^{2} \rightarrow \mathbb{R} P^{3}$ of degree 3 are planarizations. However, some of them are (e.g., dual planarizations to generic quadratic rational maps). Thus, Theorem 1.1 does not give a complete classification of all planarizations. It remains to describe cubic rational planarizations. I think that this is an interesting open problem.

Now consider a linear system $\mathcal{L}$ of conics in $\mathbb{R} P^{2}$. By definition, any conic from $\mathcal{L}$ is given by an equation of the form

$$
\lambda_{0} \phi_{0}+\cdots+\lambda_{n} \phi_{n}=0
$$

where $\phi_{0}, \ldots, \phi_{n}$ are linearly independent real quadratic forms in homogeneous coordinates of $\mathbb{R} P^{2}$, and $\lambda_{0}, \ldots, \lambda_{n}$ are some real numbers. The latter are only defined up to a common nonzero factor, thus they can be thought of as homogeneous coordinates in $\mathcal{L}$. The number $n$ is called the dimension of $\mathcal{L}$. Linear systems of dimension one are called pencils, those of dimension two are called nets, and those of dimension three are called webs. As an application of Theorem 1.1, we will prove the following theorem.

Theorem 1.2. Consider a linear web $\mathcal{L}$ of conics in $\mathbb{R} P^{2}$. Let $f: U \rightarrow \mathbb{R} P^{2}$ be a sufficiently smooth map that takes lines to $\mathcal{L}$-curves. Then there exists an open subset $U^{\prime} \subset U$, on which one of the following holds:

(1) the set $f\left(U^{\prime}\right)$ is a subset of a conic from $\mathcal{L}$;

(2) the map $\left.f\right|_{U^{\prime}}$ is a local inverse of a quadratic rational map;

(3) the map $\left.f\right|_{U^{\prime}}$ is a quadratic rational map;

(4) the map $\left.f\right|_{U^{\prime}}$ is a local branch of the multi-valued map $\Phi^{-1} \circ F$, where $\Phi$, $F: \mathbb{R} P^{2} \rightarrow Q$ are quadratic rational maps to the same irreducible quadric $Q \subset \mathbb{R} P^{3}$.

It is desirable to have a description of all sufficiently smooth maps that take lines to conics. Maps that take linear pencils of lines to conics have been studied in [10].

1.4. Families of curves and Cartan's projective connections. Let $F: U \rightarrow$ $\mathbb{R} P^{3}$ be a planarization. It defines the surface $W=F(U)$ and the 2-parameter family of planar curves on $W$ that are images of lines under $F$. Similarly, given a map $f: U \rightarrow \mathbb{R} P^{2}$ taking lines to $\mathcal{L}$-curves for some linear system $\mathcal{L}$ of conics, we obtain the 2-parameter family of conics that contain the images of lines under $f$. Thus, the problems considered in this paper are closely related to the following problem: given a surface $W$ and a class of curves on $W$ (e.g., planar curves, conics, etc.), describe all 2-parameter families of curves on $W$ from the given class that are diffeomorphic to a family of lines. A desired description has to deal with two properties. The first property is that our 2-parameter family of curves is the family of geodesics of some affine (or projective) connection. If the curves in the family are represented as graphs 
of functions $y(x)$ in some local coordinate system $(x, y)$ on $W$, then this property is equivalent to $y^{\prime \prime}(x)$ being a polynomial of degree at most 3 in $y^{\prime}(x)$. The coefficients of this polynomial may depend on $x$ and $y$ but must be independent of the choice of a curve. In other words, all curves in the family must satisfy a differential equation of the form

$$
y^{\prime \prime}=A(x, y)+B(x, y) y^{\prime}+C(x, y)\left(y^{\prime}\right)^{2}+D(x, y)\left(y^{\prime}\right)^{3} .
$$

Tresse [11] found differential relations on the coefficients $A, B, C$ and $D$ that are equivalent to the existence of a local diffeomorphism that reduces the ordinary differential equation displayed above to the form $y^{\prime \prime}=0$. Elie Cartan [1] gave an invariant interpretation of these relations in terms of the curvature tensor of the normal projective connection associated with the given family of curves. Thus, the problems considered in this paper reduce to certain systems of partial differential equations. However, I am not aware of any direct analytical methods that would lead to explicit solutions of these partial differential equations.

Organization of the paper. In Section 2, we prove Theorem 1.1. As an immediate application of this theorem, we give a short proof of a theorem of Khovanskii [3] that describes all sufficiently smooth maps taking lines to circles. In Section 3, we prove Theorem 1.2.

\section{Planarizations}

Suppose that $U \subset \mathbb{R}^{2}$, and let $F: U \mapsto \mathbb{R} P^{n}$ be a planarization. Suppose that $F(U)$ lies in an affine chart of $\mathbb{R} P^{n}$ so that we can think of $F$ as a map from $U$ to $\mathbb{R}^{n}$. Suppose also that $F$ is sufficiently many times differentiable in $U$. Fix a point $a \in U$. Let $j_{a}^{n-1} F: \mathbb{R}^{2} \rightarrow \mathbb{R}^{n}$ be the Taylor polynomial for $F$ at $a$ of degree $n-1$. We say that $F$ is nondegenerate at the point $a$ if for some line $L$ such that $L \ni a$ there is a unique hyperplane containing the set $j_{a}^{n-1} F\left(L \cap \mathbb{R}^{2}\right)$ (note that the polynomial $j_{a}^{n-1} F$ is defined on all $\left.\mathbb{R}^{2}\right)$. Clearly, in this case the set $F(U \cap L)$ is contained in the same hyperplane. It is easy to check that the notion of nondegeneracy does not depend on the choice of an affine chart. If $F$ is nondegenerate at least at one point, then the dual planarization $\hat{F}$ is nonempty.

Proposition 2.1. Suppose that $F$ is nondegenerate at a point a $\in U$. Then the restriction of $\hat{F}$ to $L_{a} \cap \hat{U}$ is a rational map of degree at most $\frac{n(n-1)}{2}$.

Proof. We can assume that $U$ lies in an affine chart and that $a$ is the origin. Let $u, v$ be affine coordinates on $U$. Then $j_{a}^{n-1} F$ has the form

$$
j_{a}^{n-1} F(u, v)=\sum_{i+j \leq n-1} u^{i} v^{j} A_{i, j}, \quad A_{i, j}=\frac{1}{i ! j !} \frac{\partial^{i+j} F}{\partial u^{i} \partial v^{j}}(0,0)
$$

by the Taylor formula. Consider a line $L=L_{\hat{a}}$, where $\hat{a} \in \hat{U}$. Suppose that, in the coordinates $(u, v)$, it is given by the equation $v=\lambda u$, where $\lambda$ is a constant (the slope of $L)$. Then the restriction of $j_{a}^{n-1} F$ to $L$ is parameterized by $u$ as follows:

$$
j_{a}^{n-1} F(u, \lambda u)=\sum_{i+j \leq n-1} \lambda^{j} u^{i+j} A_{i, j}=\sum_{\ell=0}^{n-1} u^{\ell} B_{\ell}(\lambda), \quad B_{\ell}(\lambda)=\sum_{j=0}^{\ell} \lambda^{j} A_{\ell-j, j} .
$$


Note that $B_{\ell}(\lambda)$ is a polynomial of degree $\leq \ell$. Consider the embedding $\iota: \mathbb{R}^{n} \rightarrow \mathbb{R}^{n+1}$ given by the formula $x \mapsto(x, 1)$. Then the $(n-1)$-plane $\Pi(\lambda)$ containing the set $j_{a}^{n-1} F\left(L \cap \mathbb{R}^{2}\right)$ consists of all points $x \in \mathbb{R}^{n}$ such that $\iota(x) \wedge \Omega(\lambda)=0$, where

$$
\Omega(\lambda)=\iota\left(B_{0}\right) \wedge \iota\left(B_{1}\right) \wedge \cdots \wedge \iota\left(B_{n-1}\right) .
$$

The $n$-vector $\Omega(\lambda)$ depends on $\lambda$ polynomially of degree at most $0+1+\cdots+(n-1)=$ $\frac{n(n-1)}{2}$ and is not identical zero.

Suppose that $F$ is nondegenerate at least at one point. Then there is an open set, at every point of which $F$ is nondegenerate. Therefore, the dual map $\hat{F}$ becomes rational of degree $\leq \frac{n(n-1)}{2}$ when restricted to any line in some open set of lines. We have the following statement about such maps, which generalizes well-known facts about maps, whose restrictions to lines are polynomials.

Proposition 2.2. Let $V \subset \mathbb{R} P^{2}$ be an open subset, and $f: V \rightarrow \mathbb{R}$ a function, for which there exists an open set of lines $L \subset \mathbb{R} P^{2}$ such that $L \cap V \neq \varnothing$ and $\left.f\right|_{L \cap V}$ is a rational function of degree $d$. Then the function $f$ is also a rational function of degree $d$, possibly after restriction to a smaller open set.

Proof. It suffices to assume that $V \subset \mathbb{R}^{2}$. Let $\mathcal{U}$ be the open set of lines such that, for every $L \in \mathcal{U}$, we have $V \cap L \neq \varnothing$, and $\left.f\right|_{V \cap L}$ is a rational function of degree $d$. Let $u$ and $v$ be affine coordinates on $\mathbb{R}^{2}$. We can assume that $\mathcal{U}$ contains horizontal lines $v=$ const passing through all points of $V$, and vertical lines $u=$ const passing through all points of $V$ (we may need to pass from $V$ and $\mathcal{U}$ to smaller open subsets and make an affine coordinate change to arrange this). Choose $2 d+1$ different real numbers $u_{0}, \ldots, u_{2 d}$. Note that, if $\phi(u)$ and $\psi(u)$ are degree $\leq d$ rational functions of $u$ such that $\phi\left(u_{i}\right)=\psi\left(u_{i}\right)$ for all $i=0, \ldots, 2 d$, then $\phi=\psi$. This follows from the fact that a polynomial of degree at most $2 d$ vanishing at $2 d+1$ different points must vanish identically.

Consider a rational function $g_{c}$ of degree $\leq d$ such that $g_{c}\left(u_{i}\right)=f\left(u_{i}, c\right)$ for all $i=$ $0, \ldots, 2 d$. Such a rational function exists and coincides with the function $u \mapsto f(u, c)$ by the remark just made. On the other hand, the system of equations $g_{c}\left(u_{i}\right)=f\left(u_{i}, c\right)$ can be solved for the coefficients of $g_{c}$. Note that, after we multiply both parts of the equation $g_{c}\left(u_{i}\right)=f\left(u_{i}, c\right)$ by the denominator of $g_{c}$ evaluated at $u_{i}$, we obtain a linear equation. Thus the system of equations $g_{c}\left(u_{i}\right)=f\left(u_{i}, c\right)$ is equivalent to some overdetermined linear system. At least locally, the solution of this system (suitably normalized - note that all equations are homogeneous, and the rational function does not change when we multiply all its coefficients by the same number) is given by a rational function of $u_{i}$ and $f\left(u_{i}, c\right)$. This follows from the Cramer rule. What is important for us is that the coefficients of the rational function $g_{c}$ depend rationally on $c$ ! It follows that $f$ is a rational function since $f(u, v)=g_{v}(u)$.

It remains to prove that the degree of $f$ is at most $d$. Suppose that $f$ is a rational function of degree $d^{\prime}>d$ represented as a ratio of two relatively prime polynomials, at least one of which has degree $d^{\prime}$. Then the restrictions of this polynomial to all lines in some Zariski open set of lines are polynomials of degree $d^{\prime}$. Moreover, the restrictions of two relatively prime polynomials to any line in some Zariski open set of lines are relatively prime. Thus, the restrictions of $f$ to all lines in some Zariski open set of lines will be rational functions of degree $d^{\prime}$, a contradiction. 
Propositions 2.1 and 2.2 imply the following:

Theorem 2.3. Suppose that $F: U \rightarrow \mathbb{R} P^{n}$ is a planarization that is nondegenerate at some point. Then the dual planarization $\hat{F}: \hat{U} \rightarrow \mathbb{R} P^{n *}$ is rational of degree at most $\frac{n(n-1)}{2}$ (possibly after restriction to some open subset of $\hat{U}$ ).

We now discuss the case $n=3$.

Proposition 2.4. Let $U \subset \mathbb{R}^{2}$ be an open subset. Consider a planarization $F: U \rightarrow$ $\mathbb{R}^{3}$. If there is no point of $U$, at which $F$ is nondegenerate, then the planarization $F$ is trivial, possibly after restriction to a smaller open set.

Proof. If the differential $d F$ of $F$ vanishes everywhere on $U$, then $F$ is locally constant on $U$, in particular, the restriction of $F$ to any connected component of $U$ is a trivial planarization. Suppose that $d F \neq 0$ at least at one point. Then there is an open set $U^{\prime} \subset U$ of points and an open set $V^{\prime}$ of vectors in $\mathbb{R}^{2}$ such that $d_{a} F(\xi) \neq 0$ for every $a \in U^{\prime}$ and $\xi \in V^{\prime}$.

Since the map $F$ is degenerate at all points of $U^{\prime}$, we have $d_{a} F(\xi) \wedge d_{a}^{2} F(\xi, \xi)=0$ for all points $a \in U^{\prime}$ and all vectors $\xi \in V^{\prime}$. For $a \in \mathbb{R}^{2}$ and $\xi \in V^{\prime}$, we set $X(t)=$ $F(a+t \xi)$. We have $\dot{X}(t) \neq 0$ whenever $a+t \xi \in U^{\prime}$. On the other hand, $\ddot{X} \wedge \dot{X}=0$. It follows that the $X$-image of any interval $\left(t_{1}, t_{2}\right)$ such that $a+t \xi \in U^{\prime}$ for all $t \in\left(t_{1}, t_{2}\right)$ lies in a line. Hence, if $U^{\prime \prime}$ is any convex open subset of $U^{\prime}$, then $F\left(U^{\prime \prime} \cap L\right)$ lies in a line for every line $L$ of the form $\{a+t \xi \mid t \in \mathbb{R}\}$, where $\xi \in V^{\prime}$. The set of such lines is an open set. It now follows by the Möbius theorem that, for some open subset $U^{\prime \prime \prime} \subset U$, either the set $F\left(U^{\prime \prime \prime}\right)$ is a subset of a line, or $\left.F\right|_{U^{\prime \prime \prime}}$ is a projective embedding. In both cases, $F$ is trivial on $U^{\prime \prime \prime}$.

Proof of Theorem 1.1. If there is no point, at which $F$ is nondegenerate, then by Proposition 2.4, there is an open subset of $U$, on which $F$ is a trivial planarization. Suppose now that $F$ is nondegenerate somewhere. Then the dual planarization $\hat{F}$ is defined on some open subset $\hat{U} \subset \mathbb{R} P^{2 *}$. If there is no point, at which $\hat{F}$ is nondegenerate, then, again by Proposition 2.4, there is an open subset of $\hat{U}$, on which $\hat{F}$ is a trivial planarization. It follows that $F$ is a co-trivial planarization (on an open subset of $U$ ). Finally, suppose that $\hat{F}$ is nondegenerate at some point. Then the restriction of $F$ to some open subset of $U$ coincides with the dual of $\hat{F}$, hence, by Theorem 2.3, it is a rational map of degree at most three.

Let us now discuss some immediate applications of Theorem 1.1. The following result is essentially equivalent to the theorem of Khovanskii [3]:

Theorem 2.5. Let $U$ be an open subset of $\mathbb{R} P^{2}$, and $f: U \rightarrow S^{2}$ a sufficiently smooth map such that the image $f(U \cap L)$ lies in a circle for every line $L \subset \mathbb{R} P^{2}$. Then, possibly after restriction to a smaller open set, one of the following holds:

(1) the image of $f$ lies in a circle;

(2) the map $f$ is a co-trivial planarization;

(3) the map $f$ is a quadratic rational map.

Note that circles in $S^{2}$ are precisely intersections of $S^{2}$ with two-dimensional (2D) planes. Using the methods developed above, we give a new proof of this theorem. The following lemmas are left as (simple) exercises: 
Lemma 2.6. Suppose that $P, Q$ and $R$ are cubic polynomials in one variable $t$ with complex coefficients such that $P Q=R^{2}$. Then $P, Q$ and $R$ have a nonconstant common divisor in $\mathbb{C}[t]$.

Lemma 2.7. Any rational map of $\mathbb{C} P^{1}$ to $\mathbb{C} P^{n}$ of degree at most three, whose image lies in an irreducible conic, is in fact a quadratic rational map.

Note that Lemma 2.7 applies also to rational maps of $\mathbb{R} P^{1}$ to $\mathbb{R} P^{n}$ since any such map can be complexified.

Lemma 2.8. Consider a rational map $F: \mathbb{C} P^{2} \rightarrow \mathbb{C} P^{n}$ of degree at most three such that, for a Zariski dense set of lines $L \subset \mathbb{C} P^{2}$, the image $F(L)$ lies in a plane algebraic curve of degree at most two. Then $F$ is a rational map of degree at most two, unless $F\left(\mathbb{C} P^{2}\right)$ is contained in a line.

Proof. Suppose that, for at least one line $L \subset \mathbb{C} P^{2}$ (hence for a Zariski open and dense set of lines), the set $F(L)$ lies in an irreducible conic. The map $F$ restricted to any such line must be a rational map of degree at most 2 by Lemma 2.7. Hence by Proposition 2.2 (more precisely, an obvious complex analog of it and Theorem 2.3), the map $F$ is a rational map of degree at most two. If, for every $L$, the set $F(L)$ lies in a line, then by the Möbius theorem, $F$ is a projective transformation, i.e., a rational map of degree 1 (unless $F\left(\mathbb{C} P^{2}\right)$ is contained in a line).

Proof of Theorem 2.5. If the planarization $f$ is trivial, then we have case (1) of the theorem. Suppose that $f$ is not trivial. By Theorem 1.1, it can be either co-trivial or rational of degree at most 3. In the latter case, by Lemma 2.8, the map $f$ is a rational map of degree at most two. If $f$ were a projective map, then $f$ would be a trivial planarization. Therefore, $f$ is a quadratic rational map.

\section{Linear webs of conics}

Consider a linear system $\mathcal{L}$ of conics in $\mathbb{R} P^{2}$. The linear system $\mathcal{L}$ defines a quadratic rational map $\Phi_{\mathcal{L}}: \mathbb{R} P^{2} \rightarrow \mathbb{R} P^{n}$, where $n$ is the dimension of $\mathcal{L}$. Fix a system $\left[\lambda_{0}: \cdots\right.$ : $\lambda_{n}$ ] of homogeneous coordinates in $\mathcal{L}$, then the conic with homogeneous coordinates $\left[\lambda_{0}: \cdots: \lambda_{n}\right]$ is given by the equation

$$
\lambda_{0} \phi_{0}+\cdots+\lambda_{n} \phi_{n}=0
$$

where $\phi_{0}, \ldots, \phi_{n}$ are fixed linearly independent homogeneous quadratic polynomials in the homogeneous coordinates on $\mathbb{R} P^{2}$. The map $\Phi_{\mathcal{L}}$ is defined by the equation

$$
\Phi_{\mathcal{L}}\left[x_{0}: x_{1}: x_{2}\right]=\left[\phi_{0}\left[x_{0}: x_{1}: x_{2}\right]: \cdots: \phi_{n}\left[x_{0}: x_{1}: x_{2}\right]\right]
$$

Consider first the case $n=2$, i.e., a linear net of conics.

Proposition 3.1. Suppose that $U \subset \mathbb{R} P^{2}$ is an open set, and $f: U \rightarrow \mathbb{R} P^{2}$ is a sufficiently smooth map that takes lines to $\mathcal{L}$-curves for a linear net $\mathcal{L}$ of conics. Then either the image of $f$ lies in a conic from $\mathcal{L}$, or $f$ is a local inverse of some quadratic rational map (namely, of the map $\Phi_{\mathcal{L}}$ post-composed with some Möbius transformation), possibly after restriction to a smaller open set. 
Proof. The map $\Phi_{\mathcal{L}} \circ f: U \rightarrow \mathbb{R} P^{2}$ takes lines to lines. Therefore, by the theorem of Möbius, the map $\Phi_{\mathcal{L}} \circ f$ is a restriction of a projective transformation $P: \mathbb{R} P^{2} \rightarrow \mathbb{R} P^{2}$ (unless the image of $\Phi_{\mathcal{L}} \circ f$ lies in a line, i.e., the image of $f$ lies in a conic). It follows that $f$ is a local branch of $\Phi_{\mathcal{L}}^{-1} \circ P=\left(P^{-1} \circ \Phi_{\mathcal{L}}\right)^{-1}$.

We now proceed with the proof of Theorem 1.2. Let $\mathcal{L}$ be a linear web of conics defined by linearly independent homogeneous quadratic polynomials $\phi_{0}, \phi_{1}, \phi_{2}$ and $\phi_{3}$ in the homogeneous coordinates of $\mathbb{R} P^{2}$. The map $f$ gives rise to the planarization $F=\Phi_{\mathcal{L}} \circ f: U \rightarrow \mathbb{R} P^{3}$.

Let $S$ denote the Zariski closure in $\mathbb{C} P^{3}$ of the set $\Phi_{\mathcal{L}}\left(\mathbb{C} P^{2}\right)$. This is an irreducible algebraic surface of degree at most 4 (if $S$ were a curve, then it would be a conic, but we know that $S$ is not a subset of a plane). The estimate on the degree follows from the elimination theory. If the degree of $S$ is two, then we have case (4) of Theorem 1.2, since $F$ must be a quadratic rational map by Lemma 2.8, and $f=\Phi_{\mathcal{L}}^{-1} \circ F$.

Lemma 3.2. Suppose that the degree of $S$ is at least 3. Then the map $\Phi_{\mathcal{L}}$ is a bi-rational isomorphism between $\mathbb{R} P^{2}$ and $\Phi_{\mathcal{L}}\left(\mathbb{R} P^{2}\right)$.

Proof. It suffices to prove that $\Phi_{\mathcal{L}}: \mathbb{C} P^{2} \rightarrow S$ is a bi-rational isomorphism, since $\Phi_{\mathcal{L}}^{-1}$ will then be automatically defined over real numbers.

Take a generic line $L \subset \mathbb{C} P^{2}$. The image $\Phi_{\mathcal{L}}(L)$ is an irreducible conic $C$ (otherwise $\Phi_{\mathcal{L}}$ takes lines to lines, hence by the Möbius theorem $\Phi_{\mathcal{L}}\left(\mathbb{R} P^{2}\right)$ is a planar set, a contradiction with the assumption that $\phi_{i}$ are linearly independent). Let $P_{L}$ be the 2D plane containing $C$. If $P_{L} \cap S=C$ set-theoretically, then $C$ consists of tangency points between $S$ and $P_{L}$.

First assume that this happens for all lines $L$. It follows in this case that every point $x$ of $S$ is singular, which contradicts irreducibility. Indeed, if $x \in S$ is nonsingular, then there is only one plane $P$ through $x$ such that $P$ is tangent to $S$ at $x$. On the other hand, all planes $P_{L}$ with $\Phi_{\mathcal{L}}(L) \ni x$ must have this property. It follows that all these planes coincide, and that $\Phi_{\mathcal{L}}\left(\mathbb{C} P^{2}\right)$ is a planar set, a contradiction.

Thus we can assume that $P_{L} \cap S$ is strictly bigger than the conic $C=\Phi_{\mathcal{L}}(L)$ for some $L$. Since $\Phi_{\mathcal{L}}^{-1}\left(P_{L}\right)$ is a conic containing $L$, this conic is the union of $L$ and a line $L^{\prime} \neq L$. The restriction of $\Phi_{\mathcal{L}}$ to each of the two lines $L, L^{\prime}$ has topological degree one, and the images of these two lines are different. Hence, the topological degree of $\Phi_{\mathcal{L}}$ is one.

Lemma 3.3. Suppose that a rational map $f: \mathbb{R} P^{2} \rightarrow \mathbb{R} P^{2}$ takes lines to conics. Then $f$ has degree at most two, unless $f\left(\mathbb{R} P^{2}\right)$ is a subset of a conic.

Proof. Replace $f$ with its complexification $f: \mathbb{C} P^{2} \rightarrow \mathbb{C} P^{2}$. It also takes lines to conics. Suppose that $f$ is a rational map of degree $>2$. Then the restriction of $f$ to a generic line is many-to-one. Indeed, any rational map from $\mathbb{C} P^{1}$ to a conic is the composition of a rational endomorphism of $\mathbb{C} P^{1}$ and a quadratic rational parameterization of the conic. This contradicts the fact that a generic point has finitely many preimages under $f$.

Proof of Theorem 1.2. By Theorem 1.1, there exists an open subset $U^{\prime} \subset U$ such that the map $\left.F\right|_{U^{\prime}}$ is trivial, or co-trivial, or rational of degree at most 3 . If $\left.F\right|_{U^{\prime}}$ is a trivial planarization, then $f\left(U^{\prime}\right)$ is a subset of a conic from $\mathcal{L}$, i.e., case $(1)$ of 
the theorem takes place. If $\left.F\right|_{U^{\prime}}$ is a co-trivial planarization, then, by definition of $F$, there is a linear net $\mathcal{L}^{\prime} \subset \mathcal{L}$ such that $f$ takes all lines to $\mathcal{L}^{\prime}$-curves. In this case, by Proposition 3.1, the map $f$ is a local inverse of some quadratic rational map, i.e., case (2) or the theorem takes place.

Suppose now that $\left.F\right|_{U^{\prime}}$ is a rational map. If the degree of $S$ is two, then case (4) of the theorem takes place. Otherwise, since $\Phi_{\mathcal{L}}^{-1}: \Phi_{\mathcal{L}}\left(\mathbb{R} P^{2}\right) \rightarrow \mathbb{R} P^{2}$ is a bi-rational map by Lemma 3.2, the composition $\left.f\right|_{U^{\prime}}=\left.\Phi_{\mathcal{L}}^{-1} \circ F\right|_{U^{\prime}}$ is a rational map. Since it takes lines to conics, it follows from Lemma 3.3 that $\left.f\right|_{U^{\prime}}$ is in fact a quadratic rational map, i.e., case (3) of the theorem takes place.

\section{Acknowledgments}

I am grateful to A. Khovanskii for getting me interested in the subject, and to J. Bernstein, who suggested to replace the system of all circles with an arbitrary linear system of conics. I am also grateful to L. Sukhanov for an example that led to a correction in Theorem 1.2.

This study was partially supported by the Deligne fellowship, the Simons-IUM fellowship, RFBR grants 10-01-00739-a, 11-01-00654-a, MESRF grant MK-2790.2011.1, and AG Laboratory NRU-HSE, MESRF grant ag. 11 11.G34.31.0023.

This study comprises research findings from the project "Transformations between different geometric structures", research grant No 11-01-0159, carried out within The National Research University Higher School of Economics' Academic Fund Program in $2012 / 2013$.

\section{References}

[1] E. Cartan, Sur les variétés à connexion projective, Bull. Soc. Math. 52 (1924), 205-241.

[2] F.-A. Izadi, On rectification of circles and an extension of Beltrami's theorem, Rocky mountain J. Math. 34(3) (2005), 881-899.

[3] A.G. Khovanskii, Rectification of circles, Siberian Math. J. 21 (1980), 649-652.

[4] G.S. Khovanskii, Foundations of nomography, Nauka, Moscow 1976.

[5] A.F. Möbius, Der barycentrische Calcul, 1827, in August Ferdinand Möbius, gesammelte Werke.

[6] V. Timorin, Rectification of circles and quaternions, Michigan Math. J. 51 (2003), 153-167.

[7] —, Circles and Clifford algebras, Funct. Anal. Appl. 38(1) (2004), 45-51.

[8] —- Circles and quadratic maps between spheres, Geom. Dedicata 115 (2005), 19-32.

[9] - Maps that take lines to circles, in dimension 4, Funct. Anal. Appl. 40(2) (2006), $108-116$.

[10] - Rectifiable pencils of conics, Moscow Math. J. 7(3) (2007), 561-570.

[11] A. Tresse, Détermination des invariants ponctuels de L'équations différentielle ordinaire du second ordre $y^{\prime \prime}=\omega\left(x, y, y^{\prime}\right)$, (S. Hirkel ed.), Leipzig 1896.

[12] K.G.C. von Staudt, Geometrie der lage, Nürnberg 1847.

Faculty of Mathematics and Laboratory of Algebraic Geometry, National Research University Higher School of Economics, 7 Vavilova St, 112312 Moscow, Russia

Independent University of Moscow, Bolshoy Vlasyevskiy Pereulok 11, 119002 Moscow, RUSSIA

E-mail address: vtimorin@hse.ru 
